\title{
High dielectric constant and wide band gap inverse silver oxide phases of the ordered ternary alloys of $\mathrm{SiO}_{2}, \mathrm{GeO}_{2}$, and $\mathrm{SnO}_{2}$
}

\author{
C. Sevik* and C. Bulutay ${ }^{\dagger}$ \\ Department of Physics, Bilkent University, Bilkent, Ankara, 06800, Turkey \\ (Received 19 June 2006; revised manuscript received 7 September 2006; published 15 November 2006)

\begin{abstract}
High dielectric constant and wide band gap oxides have important technological applications. The crystalline oxide polymorphs having lattice constant compatibility to silicon are particularly desirable. One recently reported candidate is the inverse silver oxide phase of $\mathrm{SiO}_{2}$. A first-principles study of this system together with its isovalent equivalents $\mathrm{GeO}_{2}$ and $\mathrm{SnO}_{2}$ as well as their ternary alloys is performed. Within the framework of density functional theory both the generalized gradient approximation and local density approximation (LDA) are employed to obtain their structural properties, elastic constants, and electronic band structures. To check the stability of these materials, phonon dispersion curves are computed which indicate that $\mathrm{GeO}_{2}$ and $\mathrm{SnO}_{2}$ have negative phonon branches whereas their ternary alloys $\mathrm{Si}_{0.5} \mathrm{Ge}_{0.5} \mathrm{O}_{2}, \mathrm{Si}_{0.5} \mathrm{Sn}_{0.5} \mathrm{O}_{2}$, and $\mathrm{Ge}_{0.5} \mathrm{Sn}_{0.5} \mathrm{O}_{2}$ are all stable within the LDA possessing dielectric constants ranging between 10 and 20. Furthermore, the lattice constant of $\mathrm{Si}_{0.5} \mathrm{Ge}_{0.5} \mathrm{O}_{2}$ is virtually identical to the $\mathrm{Si}(100)$ surface. The $G W$ band gaps of the stable materials are computed which restore the wide band gap values in addition to their high dielectric constants.
\end{abstract}

DOI: 10.1103/PhysRevB.74.193201

PACS number(s): 61.50.Ah, 62.20.Dc, 63.20.Dj, 71.15.Mb

High dielectric constant and wide band gap oxides are of general interest for the next-generation gate oxides for silicon-based electronics ${ }^{1}$ and also as host matrices for nonvolatile flash memory applications. ${ }^{2}$ Amorphous oxides have been generally preferred as they are good glass formers which tend to minimize the number of dangling bonds at the interface. In this respect, polycrystalline oxides are undesirable as the grain boundaries cause higher leakage currents and possible diffusion paths for dopants. ${ }^{1}$ On the other hand, crystalline oxide grown epitaxially on silicon ${ }^{3}$ can be favorable as it will result in high interface quality provided that it is lattice-matched to $\mathrm{Si}$.

Very recently, Ouyang and $\mathrm{Ching}^{4}$ have reported a highdensity cubic polymorph of $\mathrm{SiO}_{2}$ in the inverse $\mathrm{Ag}_{2} \mathrm{O}$ structure, named by them as the $i$ phase, possessing both a high dielectric constant, as in stishovite phase, and lattice constant compatibility to the $\mathrm{Si}(100)$ face, which makes it very attractive for electronic applications. In this computational work, we continue this search for the crystalline high dielectric constant oxides with the $i$ phases of $\mathrm{GeO}_{2}$ and $\mathrm{SnO}_{2}$ as well as their ordered ternary alloys with $\mathrm{SiO}_{2}$. This pursuit is in line with the International Technology Roadmap for Semiconductors where computational synthesis of novel high dielectric materials is emphasized.5 We employ the wellestablished $a b$ initio framework based on density functional theory within the generalized gradient approximation and local density approximation using pseudopotentials and a plane-wave basis. ${ }^{6}$

The unit cell for the ordered ternary alloy $X_{0.5} Y_{0.5} \mathrm{O}_{2}$ in the inverse $\mathrm{Ag}_{2} \mathrm{O}$ structure is shown in Fig. 1. Structural and electronic properties of the $i$-phase structures under consideration have been calculated within density functional theory, ${ }^{6}$ using the plane-wave basis pseudopotential method as implemented in the ABINIT code. ${ }^{7}$ The results are obtained under the generalized gradient approximation (GGA) and local density approximation (LDA) where for the exchangecorrelation interactions we use the Teter-Pade parametrization, ${ }^{8}$ which reproduces the Perdew-Zunger parametrization $^{9}$ (which reproduces the quantum Monte Carlo electron gas data of Ceperley and Alder ${ }^{10}$ ). We tested the LDA results under two different norm-conserving Troullier- Martins ${ }^{11}$-type pseudopotentials, which were generated by Khein and Allan (KA) and Fritz Haber Institute (FHI); for either set, the $d$ electrons were not included in the valence configuration. Our calculated values for these two types of pseudopotentials were very similar. In the course of both GGA and LDA computations, the plane-wave energy cutoff and $k$-point sampling were chosen to assure a $0.001 \mathrm{eV}$ energy convergence for all $i$-phase crystals. In the case of $\mathrm{SiO}_{2}$ this demands a 130-Ry plane-wave energy cutoff and $10 \times 10 \times 10 k$-point sampling. Phonon dispersions and phonon density of states were computed by the PHON program $^{12}$ using a $2 \times 2 \times 2$ supercell of 48 atoms to construct the dynamical matrix. The required forces were extracted from ABINIT. The corrected band-gap values are computed by obtaining self-energy corrections to the DFT KohnSham eigenvalues in the $G W$ approximation. ${ }^{13}$ All parameters used during the $G W$ calculation were chosen to assure a $0.001 \mathrm{eV}$ energy convergence.

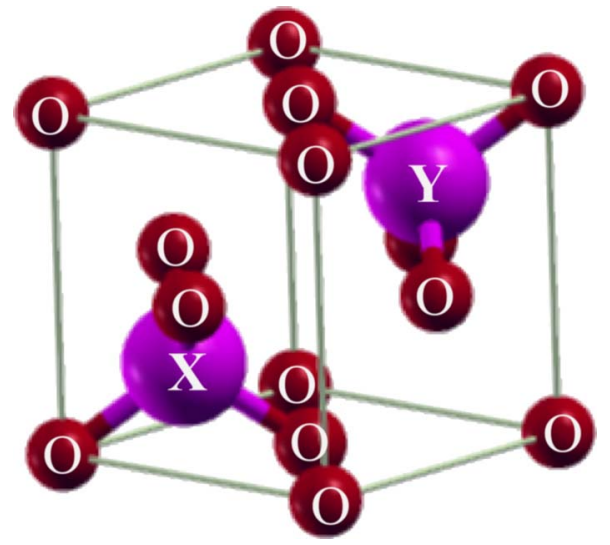

FIG. 1. (Color online) Ball and stick model of the $i$-phase-ordered ternary alloy $X_{0.5} Y_{0.5} \mathrm{O}_{2}$. 
TABLE I. First-principles LDA and GGA structural data for $i$-phase crystals.

\begin{tabular}{cccccc}
\hline \hline Crystal & & $a(\AA)$ & Density $\left(\mathrm{g} / \mathrm{cm}^{3}\right)$ & $x-\mathrm{O}(\AA)$ & $y$-O $(\AA)$ \\
\hline \multirow{2}{*}{$\mathrm{SiO}_{2}$} & LDA & 3.734 & 3.830 & 1.617 & \\
& GGA & 3.801 & 3.633 & 1.646 & \\
$\mathrm{GeO}_{2}$ & LDA & 3.916 & 5.781 & 1.696 & \\
& GGA & 4.053 & 5.215 & 1.755 & \\
$\mathrm{SnO}_{2}$ & LDA & 4.180 & 6.864 & 1.808 & \\
& GGA & 4.452 & 5.671 & 1.928 & \\
$\mathrm{Ge}_{0.5} \mathrm{Si}_{0.5} \mathrm{O}_{2}$ & LDA & 3.836 & 4.843 & 1.697 & 1.625 \\
$\mathrm{Ge}_{0.5} \mathrm{Sn}_{0.5} \mathrm{O}_{2}$ & LGA & 3.923 & 4.528 & 1.762 & 1.635 \\
& GGA & 4.042 & 6.416 & 1.688 & 1.813 \\
$\mathrm{Sn}_{0.5} \mathrm{Si}_{0.5} \mathrm{O}_{2}$ & LDA & 3.970 & 5.522 & 1.748 & 1.932 \\
& GGA & 4.114 & 5.590 & 1.818 & 1.620 \\
\hline \hline
\end{tabular}

Using $\mathrm{XO}_{2}$ and $X_{0.5} Y_{0.5} \mathrm{O}_{2}$ as the generic notation, the $\mathrm{O}$ $-X-\mathrm{O}$ and $\mathrm{O}-Y-\mathrm{O}$ bond angles are $109.47^{\circ}$ and the $X-\mathrm{O}-X$ and $X-\mathrm{O}-Y$ bond angles are $180^{\circ}$ according to the crystal construction of this cubic $i$ phase (cf. Fig. 1). Other structural information such as the lattice constants and bond lengths of all $i$-phase crystals are listed in Table I. The $\mathrm{Si}(100)$ surface lattice constant is about $3.83 \AA$; therefore, according to LDA results $\mathrm{Si}_{0.5} \mathrm{Ge}_{0.5} \mathrm{O}_{2}$ is of particular interest as it can be epitaxially grown on $\mathrm{Si}$ without any strain. According to our

(a) $\mathrm{SiO}_{2}$

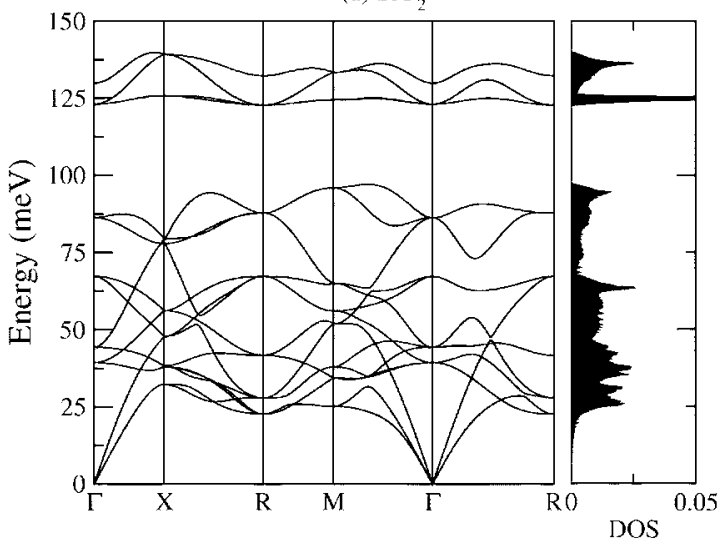

(c) $\mathrm{Ge}_{0.5} \mathrm{Sn}_{0.5} \mathrm{O}_{2}$

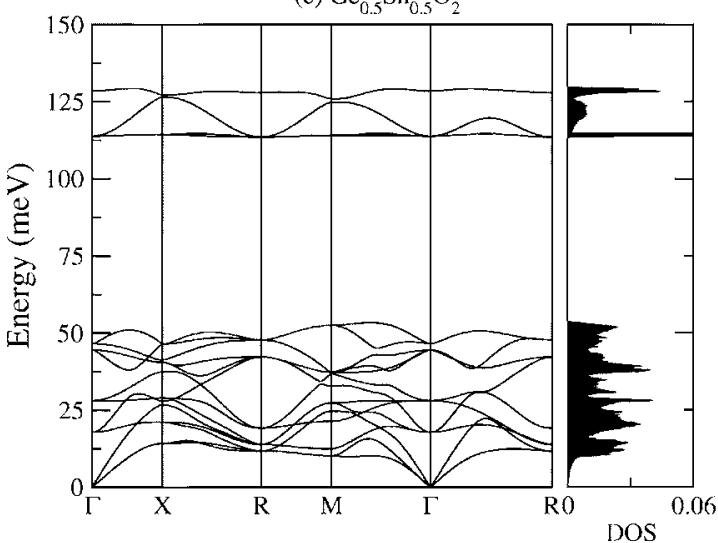

TABLE II. Elastic constants and bulk modulus for each crystal.

\begin{tabular}{cccccc}
\hline \hline Crystal & & $C_{11}(\mathrm{GPa})$ & $C_{12}(\mathrm{GPa})$ & $C_{44}(\mathrm{GPa})$ & $B(\mathrm{GPa})$ \\
\hline $\mathrm{SiO}_{2}$ & LDA & 383.6 & 260.0 & 243.0 & 301 \\
& GGA & 354.3 & 232.1 & 227.9 & 273 \\
$\mathrm{GeO}_{2}$ & LDA & 297.0 & 231.2 & 175.6 & 253 \\
$\mathrm{SnO}_{2}$ & LDA & 208.9 & 185.5 & 113.9 & 193 \\
$\mathrm{Ge}_{0.5} \mathrm{Si}_{0.5} \mathrm{O}_{2}$ & LDA & 349.4 & 253.2 & 200.0 & 285 \\
& GGA & 292.8 & 203.9 & 161.8 & 234 \\
$\mathrm{Ge}_{0.5} \mathrm{Sn}_{0.5} \mathrm{O}_{2}$ & LDA & 255.4 & 210.8 & 106.3 & 226 \\
$\mathrm{Sn}_{0.5} \mathrm{Si}_{0.5} \mathrm{O}_{2}$ & LDA & 277.5 & 217.4 & 103.9 & 237 \\
& GGA & 238.3 & 183.0 & 202.8 & 201 \\
\hline \hline
\end{tabular}

well-converged calculations $\mathrm{Si}_{0.5} \mathrm{Ge}_{0.5} \mathrm{O}_{2}$ has a lower total energy compared to both $\mathrm{SiO}_{2}$ and $\mathrm{GeO}_{2}$, the latter itself being unstable as will be shown later; this can be taken as some indication of immunity to the phase separation of this ternary alloy into its binary compounds.

The LDA and GGA results of the three independent elastic constants and bulk modulus for all crystals are tabulated in Table II. An important concern is the stability of these cubic phases. The requirement of mechanical stability on the elastic constants in a cubic crystal leads to the following constraints: $C_{11}>C_{12}, C_{11}>0, C_{44}>0$, and $C_{11}+2 C_{12}>0$. The elastic constants calculated by both the LDA and GGA
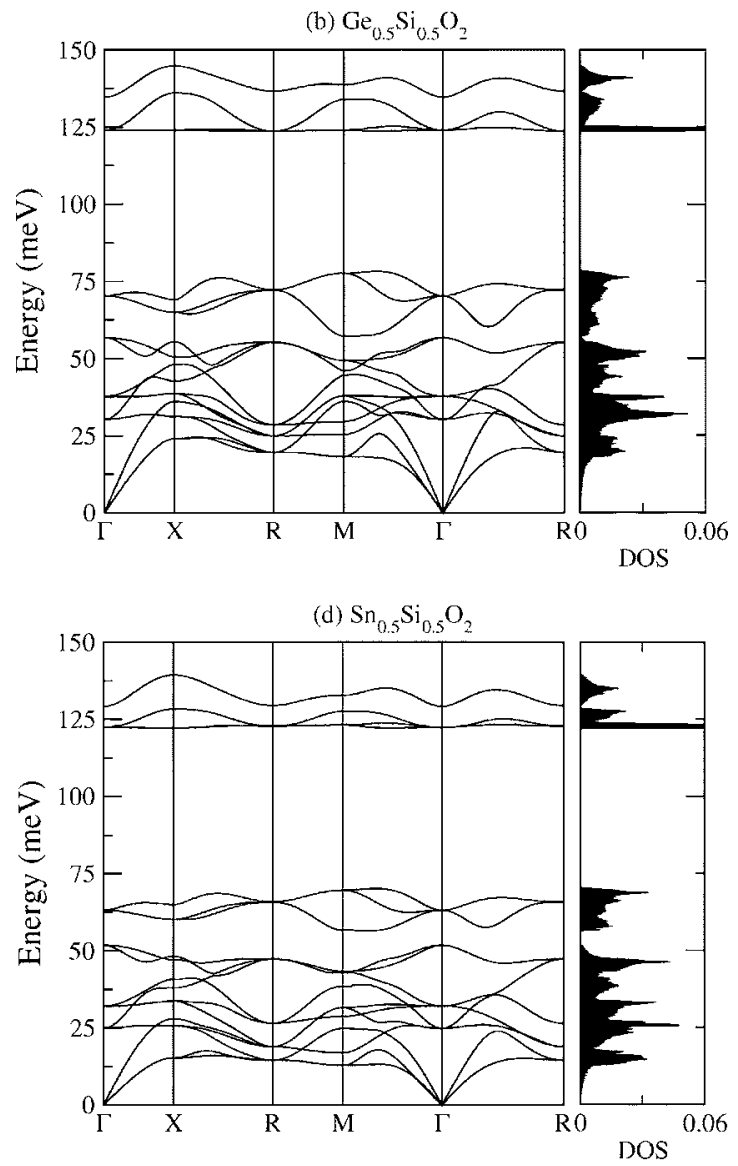

FIG. 2. LDA phonon dispersions and the phonon DOS (states/eV cell) of the stable crystals (a) $\mathrm{SiO}_{2}$, (b) $\mathrm{Ge}_{0.5} \mathrm{Si}_{0.5} \mathrm{O}_{2},(\mathrm{c}) \mathrm{Ge}_{0.5} \mathrm{Sn}_{0.5} \mathrm{O}_{2}$, and (d) $\mathrm{Si}_{0.5} \mathrm{Sn}_{0.5} \mathrm{O}_{2}$. 
TABLE III. LDA and GGA dielectric permittivity tensor for the stable crystals.

\begin{tabular}{cccc}
\hline \hline Crystal & & $\epsilon_{x x}^{0}=\epsilon_{y y}^{0}=\epsilon_{z z}^{0}$ & $\epsilon_{x x}^{\infty}=\epsilon_{y y}^{\infty}=\epsilon_{z z}^{\infty}$ \\
\hline \multirow{2}{*}{$\mathrm{SiO}_{2}$} & LDA & 9.857 & 3.285 \\
& GGA & 9.970 & 3.303 \\
$\mathrm{Ge}_{0.5} \mathrm{Si}_{0.5} \mathrm{O}_{2}$ & LDA & 11.730 & 3.416 \\
$\mathrm{Ge}_{0.5} \mathrm{Sn}_{0.5} \mathrm{O}_{2}$ & GGA & 14.383 & 3.585 \\
$\mathrm{Sn}_{0.5} \mathrm{Si}_{0.5} \mathrm{O}_{2}$ & LDA & 19.415 & 3.527 \\
& GGA & 12.883 & 3.360 \\
& & 18.096 & 3.711 \\
\hline \hline
\end{tabular}

shown in Table II satisfy these stability conditions. Furthermore, we compute the LDA and GGA phonon dispersion curves of these structures using the PHON program. ${ }^{12}$ First, to verify the validity of the results of the PHON program we compute the phonon dispersions of the $\mathrm{SiO}_{2}$ and $\mathrm{GeO}_{2}$ by using both PHON and the ANADDB extension of the ABINIT code. ${ }^{7}$ There exists good agreement between two calculations. Next, we calculate the phonon dispersions of the all $i$-phase crystals via the PHON program with forces obtained from the LDA and GGA. It is observed that $\mathrm{SiO}_{2}$ is at least locally stable whereas $\mathrm{GeO}_{2}$ and $\mathrm{SnO}_{2}$ contain negative phonon branches which signal an instability of these phases. As for their alloy $\mathrm{Ge}_{0.5} \mathrm{Sn}_{0.5} \mathrm{O}_{2}$, according to the LDA this material is stable whereas within the GGA it comes out as unstable. For the stable structures the LDA phonon dispersions and the associated phonon density of states (DOS) are shown in Fig. 2.

For the stable systems, the static and high-frequency dielectric constants are listed in Table III. The static dielectric constants falling in the range between 10 and 20 suggest that these are moderately high dielectric constant crystals. It can be observed that the GGA yields systematically higher values for the dielectric constants of these structures. Employing KA pseudopotentials, the LDA band structure for the crystals is displayed along the high-symmetry lines in Fig. 3 including the electronic DOS. The widths of the valence bands get progressively narrowed from Fig. 3(a)-3(d)-i.e., from $\mathrm{SiO}_{2}$ to $\mathrm{Sn}_{0.5} \mathrm{Si}_{0.5} \mathrm{O}_{2}$. For all of the $i$-phase crystals under consideration including the unstable ones the conduction band minima occur at the $\Gamma$ point whereas the valence band maxima are located at $R$ point making them indirect band gap semiconductors. As tabulated in Table IV, the direct the band gap values are only marginally above the indirect band
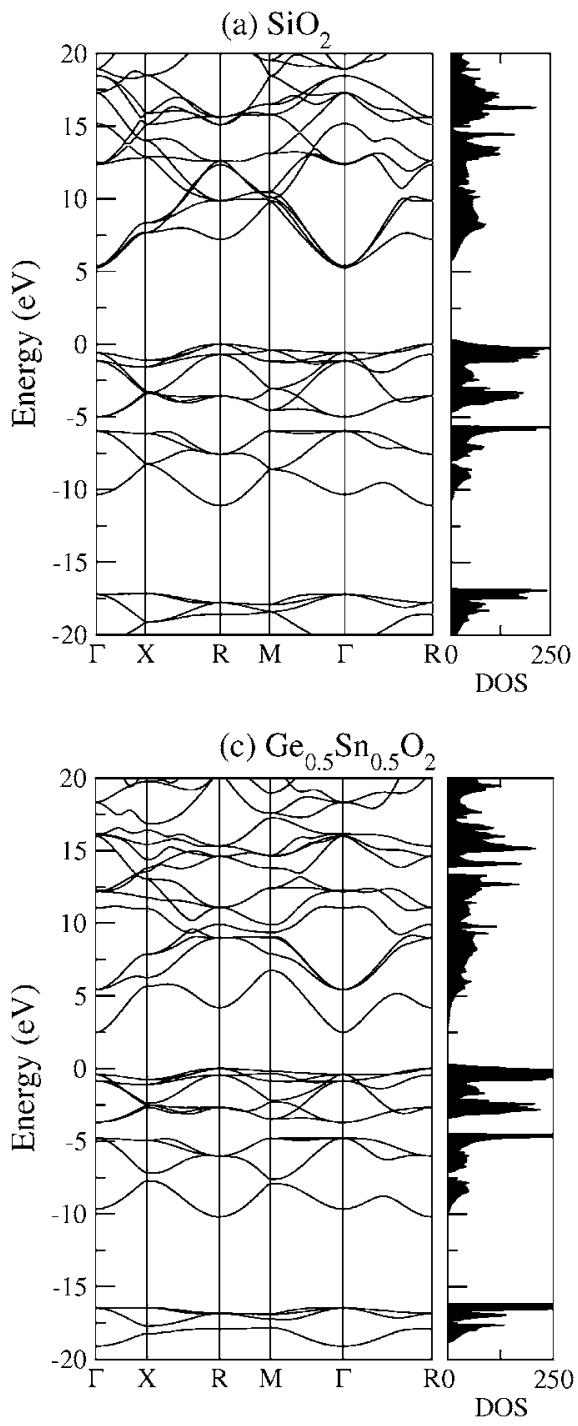
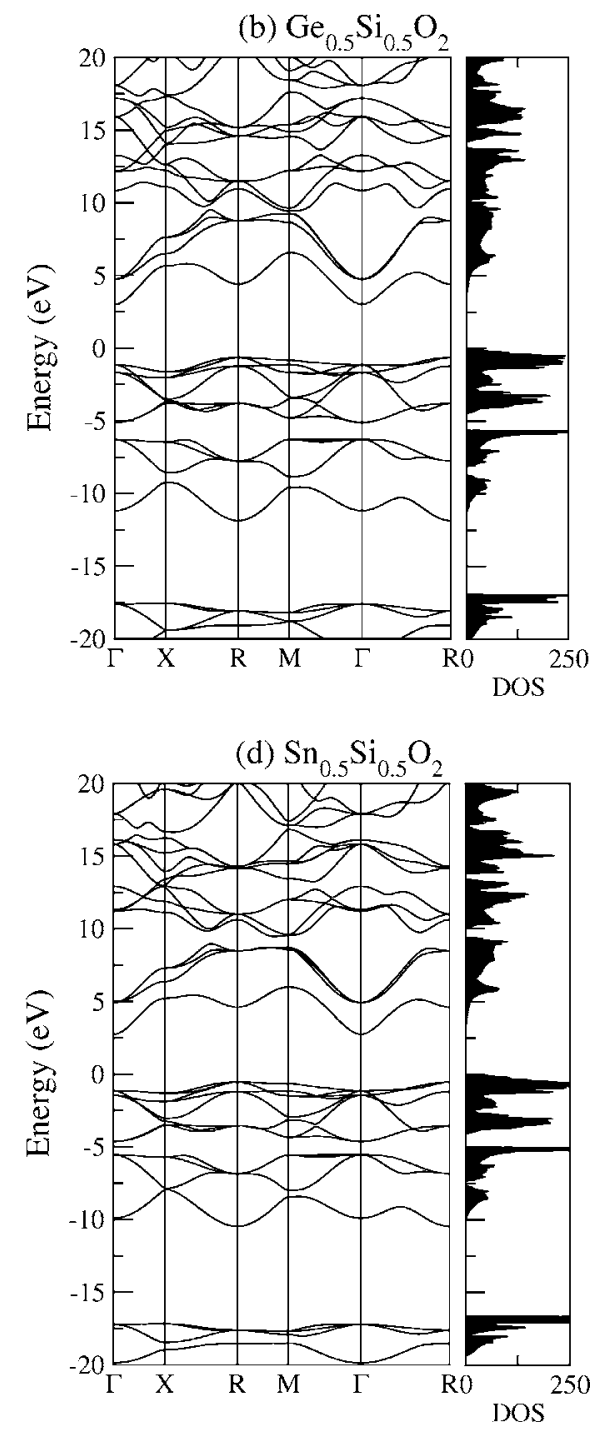

FIG. 3. LDA electronic band structure and DOS (states/eV cell) of $i$-phase (a) $\mathrm{SiO}_{2}, \quad$ (b) $\mathrm{Ge}_{0.5} \mathrm{Si}_{0.5} \mathrm{O}_{2}$, (c) $\mathrm{Ge}_{0.5} \mathrm{Sn}_{0.5} \mathrm{O}_{2}$, and (d) $\mathrm{Sn}_{0.5} \mathrm{Si}_{0.5} \mathrm{O}_{2}$. 
TABLE IV. Indirect $\left(E_{g}\right)$ and direct $\left(E_{g, \Gamma}\right)$ band gaps for each $i$-phase crystal within the LDA, GGA, and for the stable structures the $G W$ approximation (GWA).

\begin{tabular}{cccc}
\hline \hline \multicolumn{1}{c}{ Crystal } & & $E_{g}(\mathrm{eV})$ & $E_{g, \Gamma}(\mathrm{eV})$ \\
\hline $\mathrm{SiO}_{2}$ & LDA & 5.269 & 5.870 \\
& GGA & 4.584 & 5.155 \\
& GWA & 7.283 & 7.964 \\
$\mathrm{GeO}_{2}$ & LDA & 2.402 & 2.511 \\
$\mathrm{SnO}_{2}$ & LDA & 2.285 & 2.670 \\
$\mathrm{Ge}_{0.5} \mathrm{Si}_{0.5} \mathrm{O}_{2}$ & LDA & 3.666 & 4.179 \\
& GGA & 2.558 & 3.005 \\
& GWA & 5.943 & 6.513 \\
$\mathrm{Ge}_{0.5} \mathrm{Sn}_{0.5} \mathrm{O}_{2}$ & LDA & 2.487 & 2.900 \\
& GGA & 0.767 & 0.865 \\
& GWA & 4.533 & 4.972 \\
$\mathrm{Sn}_{0.5} \mathrm{Si}_{0.5} \mathrm{O}_{2}$ & LDA & 3.292 & 3.900 \\
& GGA & 1.763 & 2.304 \\
& GWA & 5.484 & 6.153 \\
\hline \hline
\end{tabular}

gap values. Again the GGA systematically yields narrower band gaps compared to the LDA.

A renown artifact of the LDA is that for semiconductors and insulators band gaps are underestimated. ${ }^{6}$ In this work, the corrected band-gap values are also provided by the $G W$ approximation. As there are different $G W$ implementations we briefly highlight the particular methodology followed in the ABINIT code. First, a converged ground-state calculation (at fixed lattice parameters and atomic positions) is done to get a self-consistent density and potential and Kohn-Sham eigenvalues and eigenfunctions at the relevant band extrema $k$ points as well as on a regular grid of $k$ points. Next, on the basis of these available Kohn-Sham data, the independentparticle susceptibility matrix $\chi_{0}$ is computed on a regular grid of $q$ points for at least two frequencies (usually, zero frequency and a large pure imaginary frequency - on the or- der of the plasmon frequency, a dozen of eV). Finally, the random phase approximation susceptibility matrix $\chi$, the dielectric matrix $\epsilon$, and its inverse $\epsilon^{-1}$ are computed. On this basis, the self-energy $\Sigma$ matrix element at the given $k$ point is computed to derive the $G W$ eigenvalues for the target states at this $k$ point. Note that this $G W$ correction is achieved as a one-shot calculation (i.e., no overall self-consistency); hence, our results technically correspond to $G_{0} W_{0}$ which has been the standard approach as originally proposal by Hedin. ${ }^{14}$ The $G W$ correction as can be observed from Table IV restores the wide band gap values; this feature is essential for these materials to provide sufficient confinement to carriers of the narrow band gap semiconductors such as silicon.

We have also considered the $i$ phase of $\mathrm{PbO}_{2}$ which turned out to be unstable and hence its ab initio data are not included. In this work, we do not consider the thermodynamic stability of these $i$-phase oxides. However, for technological applications rather than bulk systems the epitaxial growth conditions become more critical. ${ }^{15}$ A promising direction can be a finite-temperature investigation ${ }^{16}$ of these $i$-phase isovalent structures on $\operatorname{Si}(100)$ surfaces using a large number of monolayers.

This first-principles study suggests that the $i$ phases of $\mathrm{GeO}_{2}$ and $\mathrm{SnO}_{2}$ are unstable whereas $\mathrm{SiO}_{2}, \mathrm{Si}_{0.5} \mathrm{Ge}_{0.5} \mathrm{O}_{2}$, and $\mathrm{Si}_{0.5} \mathrm{Sn}_{0.5} \mathrm{O}_{2}$ are particularly promising due to their high dielectric constants as well as wide band gaps as restored by the $G W$ correction. Moreover, they are lattice-matched to the $\mathrm{Si}(100)$ face, especially for the case of $\mathrm{Si}_{0.5} \mathrm{Ge}_{0.5} \mathrm{O}_{2}$. We believe that these findings can further boost research on crystalline oxides.

This work has been supported by the European FP6 Project SEMINANO under Contract No. NMP4 CT2004 505285. We would like to thank O. Gülseren, R. Eryiğit, T. Gürel, D. Çakır, and T. Yıldırım for their useful advice. The computations were performed in part at the ULAKBIM High Performance Computing Center.
*Electronic address: sevik@fen.bilkent.edu.tr

†Electronic address: bulutay@fen.bilkent.edu.tr

${ }^{1}$ J. Robertson, Rep. Prog. Phys. 69, 327 (2006).

${ }^{2}$ D. W. Kim, T. Kim, and S. K. Banerjee, IEEE Trans. Electron Devices 50, 1823 (2003); M. She and T. J. King, ibid. 50, 1934 (2003).

${ }^{3}$ Z. Yu, Y. Liang, C. Overgaard, X. Hu, J. Curless, H. Li, Y. Wei, B. Craigo, D. Jordan, R. Droopad, J. Finder, K. Eisenbeiser, D. Marshall, K. Moore, J. Kulik, and P. Fejes, Thin Solid Films 462, 51 (2004).

${ }^{4}$ L. Ouyang and W. Y. Ching, Phys. Status Solidi B 242, R64 (2005).

${ }^{5}$ The latest edition of the ITRS roadmap can be found at http:// public.itrs.net

${ }^{6}$ R. M. Martin, Electronic Structure (Cambridge University Press, Cambridge, England, 2004).

${ }^{7}$ X. Gonze, J. M. Beuken, R. Caracas, F. Detraux, M. Fuchs, G. M.
Rignanese, L. Sindic, M. Verstraete, G. Zerah, F. Jollet, M. Torrent, A. Roy, M. Mikami, P. Ghosez, J. Y. Raty, and D. C. Allan, Comput. Mater. Sci. 25, 478 (2002).

${ }^{8}$ S. Goedecker, M. Teter, and J. Hutter, Phys. Rev. B 54, 1703 (1996).

${ }^{9}$ J. P. Perdew and A. Zunger, Phys. Rev. B 23, 5048 (1981).

${ }^{10}$ D. M. Ceperley and B. J. Alder, Phys. Rev. Lett. 45, 566 (1980).

${ }^{11}$ N. Troullier and J. L. Martins, Solid State Commun. 74, 613 1990; Phys. Rev. B 43, 1993 (1991); 43, 8861 (1991).

${ }^{12}$ D. Alfè, Computer code PHON 1998. Program available at http:// chianti.geol.ucl.ac.uk/ dario/

${ }^{13}$ W. G. Aulbur, L. Jonsson, and J. W. Wilkins, Solid State Phys. 54, 1 (2000).

${ }^{14}$ L. Hedin, Phys. Rev. 139, A796 (1965).

${ }^{15}$ K. J. Hubbard and D. G. Schlom, J. Mater. Res. 11, 2757 (1996).

${ }^{16}$ A. Pasquarello, M. S. Hybertsen, and R. Car, Nature (London) 396, 58 (1998). 